\title{
Lineability of the set of holomorphic mappings with dense range
}

\author{
JERÓNIMO LÓPEZ-SALAZAR (Madrid)
}

\begin{abstract}
Let $U$ be an open subset of a separable Banach space. Let $\mathcal{F}$ be the collection of all holomorphic mappings $f$ from the open unit disc $\mathbb{D} \subset \mathbb{C}$ into $U$ such that $f(\mathbb{D})$ is dense in $U$. We prove the lineability and density of $\mathcal{F}$ in appropriate spaces for different choices of $U$.
\end{abstract}

1. Introduction. Suppose that $U$ is an open subset of a complex Banach space $E$ and $f$ is a holomorphic mapping from the disc $\mathbb{D}=\{z \in \mathbb{C}$ : $|z|<1\}$ into $U$. Then

$$
\bigcup_{n=1}^{\infty} f\left(\frac{n}{n+1} \overline{\mathbb{D}}\right)=f(\mathbb{D}) \subset U
$$

and each subset $f\left(\frac{n}{n+1} \overline{\mathbb{D}}\right)$ is compact in $U$. By Baire's theorem, if $E$ is an infinite-dimensional Banach space, then $f(\mathbb{D})$ cannot be equal to $U$. Therefore, it seems natural to study "how big" the range of $f$ can be. In this connection, in 1973, D. Patil posed the following question at the Conference on Infinite-Dimensional Holomorphy held at University of Kentucky: if $E$ is separable, does there exist any holomorphic mapping $f$ from $\mathbb{D}$ into the open unit ball $B_{E}$ of $E$ such that $f(\mathbb{D})$ is dense in $B_{E}$ ? In 1976 , R. Aron obtained a positive answer to this problem for every separable Banach space $E$, both finite- and infinite-dimensional [1]. At the same time, J. Globevnik and W. Rudin independently obtained more general solutions:

Theorem 1.1. Let E be a separable complex Banach space.

(a) (Globevnik [5]) If $U$ is a balanced open subset of $E$, then there is a holomorphic mapping $f: \mathbb{D} \rightarrow U$ such that $f(\mathbb{D})$ is dense in $U$.

(b) (Rudin [9]) If $U$ is a connected open subset of $E$, then there is a holomorphic mapping $f: \mathbb{D} \rightarrow U$ such that $f(\mathbb{D})$ is dense in $U$. 
Using some of the ideas from those papers, we will prove the lineability and density of the set of holomorphic mappings from $\mathbb{D}$ into a Banach space with dense range. Let us recall that a collection $\mathcal{F}$ of mappings is lineable if there exists an infinite-dimensional vector space contained in $\mathcal{F} \cup\{0\}$. As is known, the aim of results on lineability is to show that some collections of special or pathological mappings are not only non-empty, but even "very large", since they contain infinitely many linearly independent elements. The first theorem on lineability is probably due to Gurariy, who proved in 1966 that there is an infinite-dimensional vector space $V$ of continuous functions on $[0,1]$ such that every $f \in V \backslash\{0\}$ is nowhere differentiable (see [6]). Let us see some other examples.

EXAMPLE 1.2.

1. The set of differentiable nowhere monotone functions from $\mathbb{R}$ into $\mathbb{R}$ is lineable [2].

2. Let $X$ be a subset of $[-\pi, \pi]$ of measure zero. The set of continuous functions on $[-\pi, \pi]$ whose Fourier series expansion is divergent at any point $t \in X$ is lineable [3].

3. The set of functions $f: \mathbb{C} \rightarrow \mathbb{C}$ such that $f(U)=\mathbb{C}$ for every open subset $U \subset \mathbb{C}$ is lineable [4].

4. If $1 \leq p<q$, then $L^{p}[0,1] \backslash L^{q}[0,1]$ is lineable [8].

5. If $p>q \geq 1$, then the sets $L^{p}(\mathbb{R}) \backslash L^{q}(\mathbb{R})$ and $\ell_{p} \backslash \ell_{q}$ are lineable [8].

2. The results. Throughout this paper, the letter $E$ will always denote a separable complex Banach space and $B_{E}$ will be the open unit ball of $E$. If $R>0$, let $D(0, R)=\{z \in \mathbb{C}:|z|<R\}$. If $U$ is a subset of $E$, the symbol $\mathcal{H}(\mathbb{D}, U)$ will denote the set of all holomorphic mappings from $\mathbb{D}$ into $E$ whose ranges are contained in $U$. The space $\mathcal{H}(\mathbb{D}, U)$ will always be endowed with the compact-open topology.

The main tool in the papers of Globevnik and Rudin was the interpolation of a dense sequence in $E$ by a sequence of holomorphic mappings on $\mathbb{D}$. We recall this result as it appears in the paper of Rudin.

Proposition 2.1 (Rudin [9]). Let $U$ be a connected open subset of $E$ and let $\left(x_{n}\right)_{n=1}^{\infty}$ be a dense sequence in $U$. Then there are three sequences, $\left(\delta_{n}\right)_{n=1}^{\infty},\left(D_{n}\right)_{n=1}^{\infty}$ and $\left(f_{n}\right)_{n=1}^{\infty}$, with the following properties:

(a) $0<2 \delta_{n+1}<\delta_{n}$ for all $n$.

(b) $D_{n}$ is an open disc centered at $e^{i / n} \in \partial \mathbb{D}$ whose radius is less than $1 / n^{2}$.

(c) Each $f_{n}$ is a continuous mapping from $\overline{\mathbb{D}}$ into $U$ and is holomorphic on $\mathbb{D}$.

(d) $f_{n}\left(e^{i / n}\right)=x_{n}$ and $f_{n}\left(e^{i / k}\right)=0$ if $n \neq k$.

(e) $\left\|f_{n}(z)\right\|<\delta_{n}$ for every $z \in \overline{\mathbb{D}} \backslash D_{n}$. 
(f) $\sum_{n=1}^{\infty} f_{n}$ is a continuous mapping from $\overline{\mathbb{D}} \backslash\{1\}$ into $U$ and is holomorphic on $\mathbb{D}$.

Using this proposition we can prove the main result in this paper.

THEOREM 2.2. The set

$$
\{f \in \mathcal{H}(\mathbb{D}, E): f(\mathbb{D}) \text { is dense in } E\}
$$

is lineable.

Proof. Let $\left(y_{m}\right)_{m=1}^{\infty}$ be a dense sequence in $E \backslash\{0\}$. Let $\left(x_{n}\right)_{n=1}^{\infty}$ be the following sequence obtained from $\left(y_{m}\right)_{m=1}^{\infty}$ :

$$
\underbrace{y_{1}}, \underbrace{y_{2}, y_{1}}, \underbrace{y_{3}, y_{2}, y_{1}}, \underbrace{y_{4}, y_{3}, y_{2}, y_{1}}, \underbrace{y_{5}, y_{4}, y_{3}, y_{2}, y_{1}} \cdots
$$

That is, $x_{n}=y_{j-k+1}$, where $j$ and $k$ are the unique natural numbers such that

$$
1 \leq k \leq j \quad \text { and } \quad n=\frac{j(j-1)}{2}+k .
$$

Let $\left(\delta_{n}\right)_{n=1}^{\infty},\left(D_{n}\right)_{n=1}^{\infty}$ and $\left(f_{n}\right)_{n=1}^{\infty}$ be sequences associated to $U=E$ and $\left(x_{n}\right)_{n=1}^{\infty}$ by Proposition 2.1. For every $k$, define $g_{k}: \overline{\mathbb{D}} \backslash\{1\} \rightarrow E$ by

$$
g_{k}=\sum_{j=k}^{\infty} f_{j(j-1) / 2+k} .
$$

Note that if $k_{1} \neq k_{2}, j_{1} \geq k_{1}$ and $j_{2} \geq k_{2}$, then

$$
\frac{j_{1}\left(j_{1}-1\right)}{2}+k_{1} \neq \frac{j_{2}\left(j_{2}-1\right)}{2}+k_{2} \text {. }
$$

Let $K$ be a compact subset of $\overline{\mathbb{D}} \backslash\{1\}$. As $e^{i / n} \rightarrow 1$ and the radius of $D_{n}$ is less than $1 / n^{2}$, there is $n_{0} \in \mathbb{N}$ such that $K \cap D_{n}=\emptyset$ for all $n \geq n_{0}$. If $z \in K$, then

$$
\sum_{n=n_{0}}^{\infty}\left\|f_{n}(z)\right\| \leq \sum_{n=n_{0}}^{\infty} \delta_{n} \leq 2 \delta_{n_{0}}<\infty .
$$

Therefore, the series which defines each function $g_{k}$ converges uniformly on compact subsets of $\overline{\mathbb{D}} \backslash\{1\}$. Hence every $g_{k}$ is continuous on $\overline{\mathbb{D}} \backslash\{1\}$ and holomorphic on $\mathbb{D}$.

Suppose that $k \in \mathbb{N}, \lambda_{1}, \ldots, \lambda_{k} \in \mathbb{C}$ and $\lambda_{1} g_{1}+\cdots+\lambda_{k} g_{k}=0$ on $\mathbb{D}$. The sum $\lambda_{1} g_{1}+\cdots+\lambda_{k} g_{k}$ is continuous on $\overline{\mathbb{D}} \backslash\{1\}$, so

$$
\begin{aligned}
0 & =\left(\lambda_{1} g_{1}+\cdots+\lambda_{k} g_{k}\right)\left(e^{\frac{i}{k(k-1) / 2+k}}\right)=\lambda_{k} f_{k(k-1) / 2+k}\left(e^{\frac{i}{k(k-1) / 2+k}}\right) \\
& =\lambda_{k} x_{k(k-1) / 2+k} .
\end{aligned}
$$

Hence $\lambda_{k}=0$ and $\lambda_{1} g_{1}+\cdots+\lambda_{k-1} g_{k-1}=0$. If we repeat this argument, we get $\lambda_{1}=\cdots=\lambda_{k}=0$. There $=$, the sequence $\left(g_{k}\right)_{k=1}^{\infty}$ is linearly independent and $\operatorname{span}\left\{g_{k}: k \in \mathbb{N}\right\}$ is $\overline{\bar{n} m}$ nite-dimensional. 
Finally, we have to prove that every non-zero element of $\operatorname{span}\left\{g_{k}: k \in \mathbb{N}\right\}$ has dense range. Pick $\alpha_{1}, \ldots, \alpha_{k} \in \mathbb{C}$, where at least $\alpha_{k}$ is non-zero. Let $x \in E$ and $\varepsilon>0$. Then there is $m$ such that

$$
\left\|y_{m}-\frac{1}{\alpha_{k}} x\right\|<\frac{\varepsilon}{2\left|\alpha_{k}\right|}
$$

Let

$$
n=\frac{(k+m-1)(k+m-2)}{2}+k .
$$

Then $x_{n}=y_{m}$ and $f_{n}$ is a summand in $g_{k}$, so

$$
g_{k}\left(e^{i / n}\right)=f_{n}\left(e^{i / n}\right)=x_{n}=y_{m} .
$$

As $f_{n}$ is not a summand in $g_{1}, \ldots, g_{k-1}$, we have

$$
g_{1}\left(e^{i / n}\right)=0, \ldots, g_{k-1}\left(e^{i / n}\right)=0 .
$$

Since $g_{1}, \ldots, g_{k}$ are continuous on $\overline{\mathbb{D}} \backslash\{1\}$, there is $w \in \mathbb{D}$ such that

$$
\left\|\left(\alpha_{1} g_{1}+\cdots+\alpha_{k} g_{k}\right)(w)-\left(\alpha_{1} g_{1}+\cdots+\alpha_{k} g_{k}\right)\left(e^{i / n}\right)\right\|<\varepsilon / 2 .
$$

Hence

$$
\begin{aligned}
\left\|\left(\alpha_{1} g_{1}+\cdots+\alpha_{k} g_{k}\right)(w)-x\right\| & <\varepsilon / 2+\left\|\left(\alpha_{1} g_{1}+\cdots+\alpha_{k} g_{k}\right)\left(e^{i / n}\right)-x\right\| \\
& =\varepsilon / 2+\left\|\alpha_{k} x_{n}-x\right\|=\varepsilon / 2+\left\|\alpha_{k} y_{m}-x\right\|<\varepsilon .
\end{aligned}
$$

This shows that $\left(\alpha_{1} g_{1}+\cdots+\alpha_{k} g_{k}\right)(\mathbb{D})$ is dense in $E$.

It is clear that the set $\mathcal{H}\left(\mathbb{D}, B_{E}\right)$ does not contain any non-zero vector space. However, the next theorem shows that the subset of $\mathcal{H}\left(\mathbb{D}, B_{E}\right)$ of holomorphic mappings with dense range has a property similar to lineability. We use $\operatorname{co}(X)$ to denote the convex hull of a subset $X$.

THEOREM 2.3. There is a linearly independent sequence $\left(h_{k}\right)_{k=1}^{\infty}$ of holomorphic mappings from $\mathbb{D}$ into $B_{E}$ such that

$$
\operatorname{co}\left(\left(h_{k}\right)_{k=1}^{\infty}\right) \subset\left\{f \in \mathcal{H}\left(\mathbb{D}, B_{E}\right): f(\mathbb{D}) \text { is dense in } B_{E}\right\} .
$$

Proof. By [1], there are holomorphic mappings $f_{1}: \mathbb{D} \rightarrow B_{c_{0}}$ and $f_{2}$ : $B_{c_{0}} \rightarrow B_{\ell_{2}}$ such that $f_{1}(\mathbb{D})$ is dense in $B_{c_{0}}$ and $f_{2}\left(B_{c_{0}}\right)$ is dense in $B_{\ell_{2}}$. We will define a sequence $\left(g_{k}\right)_{k=1}^{\infty}$ of holomorphic mappings from $\ell_{2}$ into $E$ and then $h_{k}$ will be $g_{k} \circ f_{2} \circ f_{1}$.

Denote by $\left(e_{n}\right)_{n=1}^{\infty}$ the canonical basis of $\ell_{2}$. Let $\left(y_{m}\right)_{m=1}^{\infty}$ be a dense sequence in $B_{E} \backslash\{0\}$. Let $\left(x_{n}\right)_{n=1}^{\infty}$ be the sequence

$$
\underbrace{y_{1},} \underbrace{y_{2}, y_{1}}, \underbrace{y_{3}, y_{2}, y_{1}}, \underbrace{y_{4}, y_{3}, y_{2}, y_{1}}, \underbrace{y_{5}, y_{4}, y_{3}, y_{2}, y_{1}} \cdots
$$

For each $k \in \mathbb{N}$, define $g_{k}: \ell_{2} \rightarrow E$ by

$$
g_{k}\left(\left(a_{n}\right)_{n=1}^{\infty}\right)=\sum_{n=k}^{\infty} a_{n}^{2} x_{n} .
$$


This series converges for every $\left(a_{n}\right)_{n=1}^{\infty} \in \ell_{2}$ because

$$
\sum_{n=k}^{\infty}\left\|a_{n}^{2} x_{n}\right\| \leq \sum_{n=1}^{\infty}\left|a_{n}\right|^{2}<\infty .
$$

The mapping $g_{k}$ is holomorphic on $\ell_{2}$. Indeed, for $a=\left(a_{n}\right)_{n=1}^{\infty}$ and $b=$ $\left(b_{n}\right)_{n=1}^{\infty}$ in $\ell_{2}$, let

$$
L_{a}(b)=\sum_{n=k}^{\infty} 2 a_{n} b_{n} x_{n} .
$$

Then $L_{a}: \ell_{2} \rightarrow E$ is a continuous linear mapping and

$$
\lim _{b \rightarrow 0} \frac{g_{k}(a+b)-g_{k}(a)-L_{a}(b)}{\|b\|}=0 .
$$

If $k \in \mathbb{N}$ and $\left(a_{n}\right)_{n=1}^{\infty} \in B_{\ell_{2}}$, then

$$
\left\|g_{k}\left(\left(a_{n}\right)_{n=1}^{\infty}\right)\right\| \leq \sum_{n=k}^{\infty}\left\|a_{n}^{2} x_{n}\right\| \leq \sum_{n=1}^{\infty}\left|a_{n}\right|^{2}<1 ;
$$

that is, $g_{k}\left(\left(a_{n}\right)_{n=1}^{\infty}\right) \in B_{E}$. The set $B_{E}$ is convex, so if $t_{1}, \ldots, t_{k} \in[0,1]$ and $t_{1}+\cdots+t_{k}=1$, then

$$
\left(t_{1} g_{1}+\cdots+t_{k} g_{k}\right)\left(B_{\ell_{2}}\right) \subset B_{E} .
$$

Let $t_{1}, \ldots, t_{k} \in[0,1]$ be such that $t_{1}+\cdots+t_{k}=1$. In order to prove that $\left(t_{1} g_{1}+\cdots+t_{k} g_{k}\right)\left(B_{\ell_{2}}\right)$ is dense in $B_{E}$, take $x \in B_{E}$ and $\varepsilon>0$. Then there is $m \in \mathbb{N}$ such that

$$
\left\|y_{m}-x\right\|<\varepsilon / 2 .
$$

Let $n \in \mathbb{N}$ be such that $n \geq k$ and $x_{n}=y_{m}$. Then

$$
g_{1}\left(e_{n}\right)=x_{n}=y_{m}, \quad \ldots, \quad g_{k}\left(e_{n}\right)=x_{n}=y_{m} .
$$

As $g_{1}, \ldots, g_{k}$ are continuous on $\ell_{2}$, there is $a \in B_{\ell_{2}}$ such that

$$
\left\|\left(t_{1} g_{1}+\cdots+t_{k} g_{k}\right)(a)-\left(t_{1} g_{1}+\cdots+t_{k} g_{k}\right)\left(e_{n}\right)\right\|<\varepsilon / 2 .
$$

Hence

$$
\begin{aligned}
\left\|\left(t_{1} g_{1}+\cdots+t_{k} g_{k}\right)(a)-x\right\| & <\varepsilon / 2+\left\|\left(t_{1} g_{1}+\cdots+t_{k} g_{k}\right)\left(e_{n}\right)-x\right\| \\
& =\varepsilon / 2+\left\|\left(t_{1} y_{m}+\cdots+t_{k} y_{m}\right)-x\right\| \\
& =\varepsilon / 2+\left\|y_{m}-x\right\|<\varepsilon .
\end{aligned}
$$

This shows that $\left(t_{1} g_{1}+\cdots+t_{k} g_{k}\right)\left(B_{\ell_{2}}\right)$ is dense in $B_{E}$.

For every $k$, let

$$
h_{k}=g_{k} \circ f_{2} \circ f_{1} \in \mathcal{H}(\mathbb{D}, E) .
$$

If $k \in \mathbb{N}, t_{1}, \ldots, t_{k} \in[0,1]$ and $t_{1}+\cdots+t_{k}=1$, then $\left(t_{1} h_{1}+\cdots+t_{k} h_{k}\right)(\mathbb{D})$ is densely contained in $B_{E}$ because $f_{1}(\mathbb{D})$ is dense in $B_{c_{0}}, f_{2}\left(B_{c_{0}}\right)$ is dense in $B_{\ell_{2}}$ and $\left(t_{1} g_{1}+\cdots+t_{k} g_{k}\right)\left(B_{\ell_{2}}\right)$ is dense in $B_{E}$. 
To conclude, let us prove that $\left(h_{k}\right)_{k=1}^{\infty}$ is a linearly independent sequence. Suppose that $k \in \mathbb{N}, \lambda_{1}, \ldots, \lambda_{k} \in \mathbb{C}$ and $\lambda_{1} h_{1}+\cdots+\lambda_{k} h_{k}=0$ on $\mathbb{D}$; that is,

$$
\left(\lambda_{1} g_{1}+\cdots+\lambda_{k} g_{k}\right)\left(f_{2} \circ f_{1}(z)\right)=0
$$

for all $z \in \mathbb{D}$. The set $f_{2} \circ f_{1}(\mathbb{D})$ is dense in $B_{\ell_{2}}$, so $\left(\lambda_{1} g_{1}+\cdots+\lambda_{k} g_{k}\right)(a)=0$ for all $a$ in a dense subset of $B_{\ell_{2}}$. Consequently,

$$
\begin{aligned}
\left\|\lambda_{1} x_{1}\right\| & =\left\|\left(\lambda_{1} g_{1}+\cdots+\lambda_{k} g_{k}\right)\left(e_{1}\right)\right\|=0, \\
\left\|\lambda_{1} x_{2}+\lambda_{2} x_{2}\right\| & =\left\|\left(\lambda_{1} g_{1}+\cdots+\lambda_{k} g_{k}\right)\left(e_{2}\right)\right\|=0, \\
& \vdots \\
\left\|\lambda_{1} x_{k}+\cdots+\lambda_{k} x_{k}\right\| & =\left\|\left(\lambda_{1} g_{1}+\cdots+\lambda_{k} g_{k}\right)\left(e_{k}\right)\right\|=0 .
\end{aligned}
$$

As $x_{1} \neq 0, \ldots, x_{k} \neq 0$, we deduce that $\lambda_{1}=0, \ldots, \lambda_{k}=0$.

Proposition 2.4. Let $U$ be a subset of $E$. Then

$$
\{f \in \mathcal{H}(\mathbb{D}, U): f(\mathbb{D}) \text { is dense in } U\}
$$

is a $G_{\delta}$ subset of $\mathcal{H}(\mathbb{D}, U)$.

Proof. Let $\left(y_{m}\right)_{m=1}^{\infty}$ be a dense sequence in $U$. Given $m, k \in \mathbb{N}$, let

$F_{m, k}=\left\{f \in \mathcal{H}(\mathbb{D}, U):\right.$ There is $z \in \mathbb{D}$ such that $\left.\left\|f(z)-y_{m}\right\|<1 / k\right\}$.

We will prove that each $F_{m, k}$ is an open subset of $\mathcal{H}(\mathbb{D}, U)$ and

$$
\{f \in \mathcal{H}(\mathbb{D}, U): f(\mathbb{D}) \text { is dense in } U\}=\bigcap_{m, k \in \mathbb{N}} F_{m, k} .
$$

Let $f \in F_{m, k}$. Then there is $z_{0} \in \mathbb{D}$ such that $\left\|f\left(z_{0}\right)-y_{m}\right\|<1 / k$. The set

$$
V_{f}=\left\{g \in \mathcal{H}(\mathbb{D}, U):\left\|g\left(z_{0}\right)-f\left(z_{0}\right)\right\|<1 / k-\left\|f\left(z_{0}\right)-y_{m}\right\|\right\}
$$

is a neighborhood of $f$ in $\mathcal{H}(\mathbb{D}, U)$ in the compact-open topology. If $g \in V_{f}$, then

$$
\left\|g\left(z_{0}\right)-y_{m}\right\| \leq\left\|g\left(z_{0}\right)-f\left(z_{0}\right)\right\|+\left\|f\left(z_{0}\right)-y_{m}\right\|<1 / k,
$$

so $V_{f} \subset F_{m, k}$. This shows that $F_{m, k}$ is open in $\mathcal{H}(\mathbb{D}, U)$.

To prove $(2.1)$, take $h \in \mathcal{H}(\mathbb{D}, U)$ such that $h(\mathbb{D})$ is dense in $U$. Then for every $m, k \in \mathbb{N}$ there is $z \in \mathbb{D}$ such that $\left\|h(z)-y_{m}\right\|<1 / k$; that is, $h \in \bigcap_{m, k \in \mathbb{N}} F_{m, k}$.

Conversely, suppose that $h \in \bigcap_{m, k \in \mathbb{N}} F_{m, k}$. Let $x \in U$ and $\varepsilon>0$. As $\left(y_{m}\right)_{m=1}^{\infty}$ is dense in $U$, there are $k_{0}, m_{0} \in \mathbb{N}$ such that

$$
1 / k_{0}<\varepsilon / 2 \text { and }\left\|y_{m_{0}}-x\right\|<1 / k_{0} .
$$

As $h \in F_{m_{0}, k_{0}}$, there is $z \in \mathbb{D}$ such that $\left\|h(z)-y_{m_{0}}\right\|<1 / k_{0}$. Therefore,

$$
\|h(z)-x\| \leq\left\|h(z)-y_{m_{0}}\right\|+\left\|y_{m_{0}}-x\right\|<1 / k_{0}+1 / k_{0}<\varepsilon,
$$

which shows that $h(\mathbb{D})$ is dense in $U$. This completes the proof. 
ThEOREM 2.5. The set

$$
\{f \in \mathcal{H}(\mathbb{D}, E): f(\mathbb{D}) \text { is dense in } E\}
$$

is a dense $G_{\delta}$ subset of $\mathcal{H}(\mathbb{D}, E)$.

Proof. Let $K$ be a compact subset of $\mathbb{D}, g \in \mathcal{H}(\mathbb{D}, E)$ and $\varepsilon>0$. Then there is a polynomial $P: \mathbb{C} \rightarrow E$ such that $\|P(z)-g(z)\|<\varepsilon / 2$ for all $z \in K$ (see [7, Theorem 7.11]). Then

Let $\left(y_{m}\right)_{m=1}^{\infty},\left(x_{n}\right)_{n=1}^{\infty}$ and $\left(f_{n}\right)_{n=1}^{\infty}$ be as in the proof of Theorem 2.2 .

$$
f=\sum_{n=1}^{\infty} f_{n}
$$

is a continuous mapping from $\overline{\mathbb{D}} \backslash\{1\}$ into $E$ which is holomorphic on $\mathbb{D}$. If we define

$$
t=\frac{\varepsilon}{2 \sup _{z \in K}\|f(z)\|+1},
$$

then $\|(t f+P)(z)-g(z)\|<\varepsilon$ for all $z \in K$.

In order to prove that $(t f+P)(\mathbb{D})$ is dense in $E$, take $x \in E$ and $\delta>0$. As $\left(y_{m}\right)_{m=1}^{\infty}$ is dense in $E$, there is $m \in \mathbb{N}$ such that

$$
\left\|y_{m}-\left(\frac{1}{t} x-\frac{1}{t} P(1)\right)\right\|<\frac{\delta}{3 t}
$$

that is,

$$
\left\|t y_{m}+P(1)-x\right\|<\delta / 3
$$

Since $P$ is continuous on $\mathbb{C}$ and $e^{i / n} \rightarrow 1$, there is $n_{0} \in \mathbb{N}$ such that

$$
\left\|P\left(e^{i / n}\right)-P(1)\right\|<\delta / 3 \quad \text { for every } n \geq n_{0} .
$$

Now, take $n \in \mathbb{N}$ such that $x_{n}=y_{m}$ and $n \geq n_{0}$. The mapping $t f+P$ is continuous on $\overline{\mathbb{D}} \backslash\{1\}$, so there is $w \in \mathbb{D}$ such that

$$
\left\|(t f+P)(w)-(t f+P)\left(e^{i / n}\right)\right\|<\delta / 3 .
$$

Thus,

$$
\begin{aligned}
\|(t f+P)(w)-x\| & <\delta / 3+\left\|t f\left(e^{i / n}\right)+P\left(e^{i / n}\right)-x\right\| \\
& =\delta / 3+\left\|t x_{n}+P\left(e^{i / n}\right)-x\right\| \\
& =\delta / 3+\left\|t y_{m}+P\left(e^{i / n}\right)-x\right\| \\
& \leq \delta / 3+\left\|t y_{m}+P(1)-x\right\|+\left\|P\left(e^{i / n}\right)-P(1)\right\| \\
& <\delta .
\end{aligned}
$$

This shows that $(t f+P)(\mathbb{D})$ is dense in $E$.

The proof of the density of $\left\{f \in \mathcal{H}\left(\mathbb{D}, B_{E}\right): f(\mathbb{D})\right.$ is dense in $\left.B_{E}\right\}$ in $\mathcal{H}\left(\mathbb{D}, B_{E}\right)$ is much longer and we will need some preliminary results. 
Proposition 2.6. Let $0<R<1$ and $\varepsilon>0$. There is a sequence $\left(a_{n}\right)_{n=1}^{\infty}$ of real numbers such that $0<a_{n}<1$ for all $n, \sum_{n=1}^{\infty}\left(1-a_{n}\right)<\infty$ and $|b(z)-1|<\varepsilon$ for every $z \in \bar{D}(0, R)$, where

$$
b(z)=\prod_{n=1}^{\infty} \frac{a_{n}-z}{1-a_{n} z}
$$

is the usual Blaschke product (see [10, p. 302]).

Proof. We can assume that $\varepsilon<1$. Let $n \in \mathbb{N}$ and $\delta_{n}=\varepsilon / 2^{n+1} \in(0,1)$. As $R<1$, we have $\delta_{n} R<\delta_{n}$, so

$$
1+R-\delta_{n} R>1+R-\delta_{n}>0 \text {. }
$$

This implies that

$$
\frac{1+R-\delta_{n}}{1+R-\delta_{n} R}<1
$$

Therefore, there is $a_{n} \in \mathbb{R}$ such that

$$
1-\frac{1}{2^{n}}<a_{n}<1 \text { and } \frac{1+R-\delta_{n}}{1+R-\delta_{n} R}<a_{n}<1 .
$$

The first inequality in (2.2) implies that $\sum_{n=1}^{\infty}\left(1-a_{n}\right)<\infty$, so the product

$$
b(z)=\prod_{n=1}^{\infty} \frac{a_{n}-z}{1-a_{n} z}
$$

is well defined for every $z \in \mathbb{D}$. The second property in (2.2) implies that

$$
1+R-\delta_{n}<a_{n}+a_{n} R-\delta_{n} a_{n} R,
$$

So

$$
\left(1-a_{n}\right)(1+R)<\delta_{n}\left(1-a_{n} R\right) .
$$

Let $z \in \mathbb{C}$ be such that $|z| \leq R$. Then

$$
\begin{aligned}
\left|\left(a_{n}-z\right)-\left(1-a_{n} z\right)\right| & \leq\left(1-a_{n}\right)+\left(1-a_{n}\right)|z| \\
& \leq\left(1-a_{n}\right)+\left(1-a_{n}\right) R=\left(1-a_{n}\right)(1+R) .
\end{aligned}
$$

We now use the inequalities (2.3) and (2.4):

$$
\left|\left(a_{n}-z\right)-\left(1-a_{n} z\right)\right|<\delta_{n}\left(1-a_{n} R\right) \leq \delta_{n}\left(1-a_{n}|z|\right) \leq \delta_{n}\left|1-a_{n} z\right| .
$$

Therefore,

$$
\left|\frac{a_{n}-z}{1-a_{n} z}-1\right|<\delta_{n}=\frac{\varepsilon}{2^{n+1}}
$$

for every $n \in \mathbb{N}$ and every $z \in \mathbb{C}$ such that $|z| \leq R$.

Let $z \in \bar{D}(0, R)$. For each $n$, let

$$
w_{n}=\frac{a_{n}-z}{1-a_{n} z} .
$$


Note that $\left|w_{n}\right|<1$ because $|z|<1$. In addition, there is $m \in \mathbb{N}$ such that

$$
\left|b(z)-\prod_{n=1}^{m} w_{n}\right|<\frac{\varepsilon}{2} .
$$

Then

$$
\begin{aligned}
|b(z)-1| & <\frac{\varepsilon}{2}+\left|\prod_{n=1}^{m} w_{n}-1\right| \leq \frac{\varepsilon}{2}+\left|\prod_{n=2}^{m} w_{n}-1\right|\left|w_{1}\right|+\left|w_{1}-1\right| \\
& \leq \frac{\varepsilon}{2}+\left|\prod_{n=2}^{m} w_{n}-1\right|+\left|w_{1}-1\right| \leq \cdots \leq \frac{\varepsilon}{2}+\sum_{n=1}^{m}\left|w_{n}-1\right| .
\end{aligned}
$$

By (2.5), we obtain

$$
|b(z)-1|<\frac{\varepsilon}{2}+\sum_{n=1}^{m} \frac{\varepsilon}{2^{n+1}}<\varepsilon \quad \text { for all } z \in \bar{D}(0, R) .
$$

Proposition 2.7. Let $\left(y_{m}\right)_{m=1}^{\infty}$ be a dense sequence in $B_{E}$. Then for every $m, k \in \mathbb{N}$, the set

$$
F_{m, k}=\left\{f \in \mathcal{H}\left(\mathbb{D}, \bar{B}_{E}\right): \text { There is } z \in \mathbb{D} \text { such that }\left\|f(z)-y_{m}\right\|<1 / k\right\}
$$

is dense in $\mathcal{H}\left(\mathbb{D}, \bar{B}_{E}\right)$.

Proof. Let $g \in \mathcal{H}\left(\mathbb{D}, \bar{B}_{E}\right), K$ a compact subset of $\mathbb{D}$ and $0<\varepsilon<1 /(3 k)$. We can assume that $K=\bar{D}(0, R)$ for some $0<R<1$. By Proposition 2.6, there is a sequence $\left(a_{n}\right)_{n=1}^{\infty}$ of real numbers such that $0<a_{n}<1$ for every $n$, the Blaschke product

$$
b(z)=\prod_{n=1}^{\infty} \frac{a_{n}-z}{1-a_{n} z}
$$

defines a holomorphic function on $\mathbb{D},|b(z)|<1$ for all $z \in \mathbb{D}$ and $|b(z)-1|<\varepsilon$ if $|z| \leq R$. As $b$ is uniformly continuous on $\bar{D}\left(0, a_{1}\right)$, there is $\delta>0$ such that if $z, w \in \bar{D}\left(0, a_{1}\right)$ and $|z-w|<\delta$, then

$$
|b(z)-b(w)|<\varepsilon .
$$

As before, $\left(x_{n}\right)_{n=1}^{\infty}$ will be the sequence

$$
\underbrace{y_{1}}, \underbrace{y_{2}, y_{1}}, \underbrace{y_{3}, y_{2}, y_{1}}, \underbrace{y_{4}, y_{3}, y_{2}, y_{1}}, \underbrace{y_{5}, y_{4}, y_{3}, y_{2}, y_{1}} \ldots
$$

Let $\left(\delta_{n}\right)_{n=1}^{\infty},\left(D_{n}\right)_{n=1}^{\infty}$ and $\left(f_{n}\right)_{n=1}^{\infty}$ be the sequences associated to $U=B_{E}$ and $\left(x_{n}\right)_{n=1}^{\infty}$ by Proposition 2.1. Let $n \in \mathbb{N}$ be chosen so that

$$
x_{n}=y_{m}, \quad R+1 / n^{2}<1, \quad \delta_{n}<\varepsilon, \quad 1 / n^{2}<\delta .
$$

For each $z \in \mathbb{D}$, let

$$
f(z)=b\left(a_{1} e^{-i / n} z\right) g(z)+f_{n}(z)
$$


The mapping $f$ is holomorphic from $\mathbb{D}$ into $E$. If $z \in \mathbb{D} \backslash D_{n}$, then

$$
\|f(z)\| \leq\|g(z)\|+\left\|f_{n}(z)\right\| \leq\|g(z)\|+\delta_{n} \leq 1+\delta_{n}<1+\varepsilon .
$$

If $z \in \mathbb{D} \cap D_{n}$, then $\left|z-e^{i / n}\right|<1 / n^{2}<\delta$ by Proposition 2.1(b). Hence

$$
\left|a_{1} e^{-i / n} z-a_{1}\right|=a_{1}\left|z-e^{i / n}\right|<\delta .
$$

Moreover, $a_{1} e^{-i / n} z \in \bar{D}\left(0, a_{1}\right)$. By $(2.6)$,

$$
\left|b\left(a_{1} e^{-i / n} z\right)\right|=\left|b\left(a_{1} e^{-i / n} z\right)-b\left(a_{1}\right)\right|<\varepsilon .
$$

Consequently,

$$
\|f(z)\| \leq\left|b\left(a_{1} e^{-i / n} z\right)\right|+\left\|f_{n}(z)\right\|<\varepsilon+1 .
$$

Therefore, $\|f(z)\|<1+\varepsilon$ for every $z \in \mathbb{D}$.

Let $z \in \bar{D}(0, R)$. Then $a_{1} e^{-i / n} z$ also belongs to $\bar{D}(0, R)$, so

$$
\left|b\left(a_{1} e^{-i / n} z\right)-1\right|<\varepsilon .
$$

As $R+1 / n^{2}<1$, we have $\bar{D}(0, R) \cap D_{n}=\emptyset$, so $z \in \mathbb{D} \backslash D_{n}$ and $\left\|f_{n}(z)\right\|<$ $\delta_{n}<\varepsilon$. Hence

$$
\|f(z)-g(z)\| \leq\left|b\left(a_{1} e^{-i / n} z\right)-1\right| \cdot\|g(z)\|+\left\|f_{n}(z)\right\|<2 \varepsilon
$$

for every $z \in \bar{D}(0, R)$.

Since $f_{n}$ is continuous on $\overline{\mathbb{D}}$, there is $z_{1} \in \mathbb{D} \cap D_{n}$ such that

$$
\left\|f_{n}\left(z_{1}\right)-f_{n}\left(e^{i / n}\right)\right\|<\varepsilon .
$$

Again by (2.6),

$$
\left|b\left(a_{1} e^{-i / n} z_{1}\right)\right|=\left|b\left(a_{1} e^{-i / n} z_{1}\right)-b\left(a_{1}\right)\right|<\varepsilon
$$

and

$$
\begin{aligned}
\left\|f\left(z_{1}\right)-y_{m}\right\| & \leq \varepsilon+\left\|f_{n}\left(z_{1}\right)-y_{m}\right\| \leq 2 \varepsilon+\left\|f_{n}\left(e^{i / n}\right)-y_{m}\right\| \\
& =2 \varepsilon+\left\|x_{n}-y_{m}\right\|=2 \varepsilon .
\end{aligned}
$$

Finally, we consider the function $\frac{1}{1+\varepsilon} f \in \mathcal{H}\left(\mathbb{D}, \bar{B}_{E}\right)$, which belongs to $F_{m, k}$ as

$$
\begin{aligned}
\left\|\frac{1}{1+\varepsilon} f\left(z_{1}\right)-y_{m}\right\| & =\left\|\frac{1}{1+\varepsilon} f\left(z_{1}\right)-f\left(z_{1}\right)\right\|+\left\|f\left(z_{1}\right)-y_{m}\right\| \\
& \leq \varepsilon+2 \varepsilon=3 \varepsilon<1 / k .
\end{aligned}
$$

If $z \in K=\bar{D}(0, R)$, then

$$
\begin{aligned}
\left\|g(z)-\frac{1}{1+\varepsilon} f(z)\right\| & \leq\|g(z)-f(z)\|+\left\|f(z)-\frac{1}{1+\varepsilon} f(z)\right\| \\
& <2 \varepsilon+\varepsilon=3 \varepsilon .
\end{aligned}
$$

This proves that $F_{m, k}$ is dense in $\mathcal{H}\left(\mathbb{D}, \bar{B}_{E}\right)$. 
ThEOREM 2.8. The set

$$
\left\{f \in \mathcal{H}\left(\mathbb{D}, B_{E}\right): f(\mathbb{D}) \text { is dense in } B_{E}\right\}
$$

is a dense $G_{\delta}$ subset of $\mathcal{H}\left(\mathbb{D}, B_{E}\right)$.

Proof. As before, $\left(y_{m}\right)_{m=1}^{\infty}$ denotes a dense sequence in $B_{E}$ and we set

$F_{m, k}=\left\{f \in \mathcal{H}\left(\mathbb{D}, \bar{B}_{E}\right):\right.$ There is $z \in \mathbb{D}$ such that $\left.\left\|f(z)-y_{m}\right\|<1 / k\right\}$.

In Propositions 2.4 and 2.7, we have seen that each $F_{m, k}$ is a dense open subset of $\mathcal{H}\left(\mathbb{D}, \bar{B}_{E}\right)$ and

$$
\left\{f \in \mathcal{H}\left(\mathbb{D}, \bar{B}_{E}\right): f(\mathbb{D}) \text { is dense in } \bar{B}_{E}\right\}=\bigcap_{m, k \in \mathbb{N}} F_{m, k} .
$$

The space $\mathcal{H}(\mathbb{D}, E)$, endowed with the compact-open topology, is a complete metric space. As $\mathcal{H}\left(\mathbb{D}, \bar{B}_{E}\right)$ is a closed subset of $\mathcal{H}(\mathbb{D}, E)$, it follows that $\mathcal{H}\left(\mathbb{D}, \bar{B}_{E}\right)$ is also a complete metric space. By Baire's theorem and (2.7), the set

$$
\left\{f \in \mathcal{H}\left(\mathbb{D}, \bar{B}_{E}\right): f(\mathbb{D}) \text { is dense in } \bar{B}_{E}\right\}
$$

is dense in $\mathcal{H}\left(\mathbb{D}, \bar{B}_{E}\right)$.

Finally, let $h \in \mathcal{H}\left(\mathbb{D}, B_{E}\right), K$ a compact subset of $\mathbb{D}$ and $\varepsilon>0$. Then there is $f: \mathbb{D} \rightarrow \bar{B}_{E}$ such that $f(\mathbb{D})$ is dense in $\bar{B}_{E}$ and $\|f(z)-h(z)\|<\varepsilon$ for every $z \in K$. If there were $z_{1} \in \mathbb{D}$ such that $\left\|f\left(z_{1}\right)\right\|=1$, then $\|f(z)\|=1$ for all $z \in \mathbb{D}$, so $f$ would not have dense range. Therefore, we deduce that $f \in \mathcal{H}\left(\mathbb{D}, B_{E}\right)$.

Acknowledgements. This research was started while the author was visiting the Department of Mathematics of Kent State University. The author thanks the Department for their hospitality. He especially thanks Richard Aron for his help during the preparation of this paper.

The author has been supported by Universidad Complutense de Madrid, grant $\mathrm{BE} 45 / 08$.

\section{References}

[1] R. M. Aron, The range of vector valued holomorphic mappings, Ann. Polon. Math. 33 (1976), 17-20.

[2] R. M. Aron, V. Gurariy and J. B. Seoane Sepúlveda, Lineability and spaceability of sets of functions on $\mathbb{R}$, Proc. Amer. Math. Soc. 133 (2005), 795-803.

[3] R. M. Aron, D. Pérez García and J. B. Seoane Sepúlveda, Algebrability of the set of non-convergent Fourier series, Studia Math. 175 (2006), 83-90.

[4] R. M. Aron and J. B. Seoane Sepúlveda, Algebrability of the set of everywhere surjective functions on $\mathbb{C}$, Bull. Belg. Math. Soc. Simon Stevin 14 (2007), 25-31.

[5] J. Globevnik, The range of vector-valued analytic functions, Ark. Mat. 14 (1976), $113-118$. 
[6] V. I. Gurariy, Subspaces and bases in spaces of continuous functions, Dokl. Akad. Nauk 167 (1966), 971-973 (in Russian).

[7] J. Mujica, Complex Analysis in Banach Spaces, Dover Publ., 2010.

[8] G. Muñoz Fernández, N. Palmberg, D. Puglisi and J. B. Seoane Sepúlveda, Lineability in subsets of measure and function spaces, Linear Algebra Appl. 428 (2008), 2805-2812.

[9] W. Rudin, Holomorphic maps of discs into F-spaces, in: Complex Analysis (Lexington, KY, 1976), Lecture Notes in Math. 599,Springer, 1977, 104-108.

[10] W. Rudin, Real and Complex Analysis, McGraw-Hill, 1987.

Jerónimo López-Salazar

Departamento de Análisis Matemático

Universidad Complutense de Madrid

28040 Madrid, Spain

E-mail: jlopezsalazar@mat.ucm.es 